\title{
MTBF (Metric That Betrays Folk)
}

\author{
Cristian MAIORANO \\ Ansaldo STS France, 4 Avenue du Canada, 91940 Les Ulis, France. E-mail: Cristian.maiorano@ansaldo-sts.fr \\ Emanuele PASCALE \\ Ansaldo STS Italy, Via Argine, 425, 80147 Napoli, Italy. E-mail: Emanuele.pascale@ansaldo-sts.com \\ Fred SHENKELBERG \\ E-mail:fms@fmsreliability.com \\ Laurent BOUILLAUT \\ IFSTTAR/COSYS/GRETTIA - University Paris Est, Champs-sur-Marne, France E-mail : \\ laurent.bouillaut@ifsttar.fr \\ Paolo SANNINO \\ Ansaldo STS Italy, Via Argine, 425, 80147 Napoli, Italy.E-mail: paolo.sannino@ansaldo-sts.com \\ Yusmery SOLORZANO \\ Ansaldo STS Italy, Via Argine, 425, 80147 Napoli, Italy.E-mail: yusmery.solorzano@ansaldo-sts.com \\ Stanislao BORRIELLO \\ Ansaldo STS Italy, Via Argine, 425, 80147 Napoli, Italy.E-mail: stanislao.borriello@ansaldo-sts.com \\ Pietro MARMO \\ Ansaldo STS Italy, Via Argine, 425, 80147 Napoli, Italy. E-mail: pietro.marmo@ansaldo-sts.com
}

Each industrial field seems affected by revolutionary innovations that will push forward the quality and variety of disparate services: driverless trains running 24 hours/day, self-driving cars and interconnected objects are only some of the latest trends.

The intrinsic common aspect among all these promising concepts is the increase in reliability needs: human intervention for restoring and rescuing is not tolerated anymore. Despite this consideration, electronic equipment datasheets of many vendors do measure reliability through fuzzy metrics, the MTBF (Mean Time Between Failures) being the first among those.

Firstly, this paper aims to provide information on the origin of the MTBF and on the reason why it is nowadays the most common mean for referring to reliability. Furthermore, the reasons behind the fallacy of the MTBF are explained. Using MTBF as synthetic indicator of reliability does often represents the wrong choice. Indeed, high MTBF figures could be measured even if the actual reliability is low. However, MTBF use is often imposed by regulations or contracts, and some examples are reported in this paper related to the railway signaling domain. Finally, alternative structured ways of communicating about reliability, based on further concepts than the bare MTBF, are provided as conclusion of the article.

Keywords: MTBF, Reliability, Engineering, Railway, RAM, Safety, Weibull

\section{The role of reliability in railway industry}

Railway signalling domain deals with the development and deployment of equipment used to increase the efficiency of operations for both mass transit and high-speed lines. Governments, local communities and rail operators have growingly focused on solutions allowing connecting points of the same city, region or state in efficiency growing ways. Trains, tracks and stations have been filled with electronic based equipment allowing the automation of tasks previously performed by human operators. Consequently, the need of efficiency has constantly increased over the time. It is clear that the concept of "reliability" is essential within this perspective. European standards (CENELEC) taken as reference for railway signalling equipment encompass reliability concept. All the bids and contracts devote paragraphs to ensure the technical solution respects the highest reliability. However, how this concept is formulated? What are the figures to demonstrate this requirement is met? Despite CENELEC standards, such the

Proceedings of the 29th European Safety and Reliability Conference.

Edited by Michael Beer and Enrico Zio

Copyright (C2019 by ESREL2019 Organizers. Published by Research Publishing, Singapore

ISBN: 981-973-0000-00-0 :: doi: 10.3850/981-973-0000-00-0 esrel2019-paper 
latest EN50129:2018, provide clear indications about the need of reliability requirements to be met, they adopt reliability data handbooks that in several cases are built on strong assumptions: one of them is the constant failure rate over the time, and on the abuse of MTBF.

\section{What the MTBF is?}

The term MTBF stands for Mean Time Between Failures or, Mean operating Time Between Failures. Within maintenance management, it is the most important KPI after Physical Availability. Unlike MTTF (Mean Time To Failure), which relates directly to available equipment time, MTBF also adds up the time spent inside a repair. That is, it starts its count from a certain failure and only stops its counter when this fault was remedied, started and repeated itself again. MTBF can only be used for nonrepairable equipment and for repairable equipment where at each repair an as-good-asnew situation is restored.

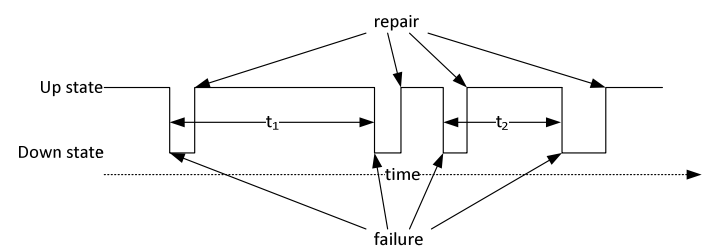

Fig. 1. Mean Time Between Failures

The MTBF in the Fig. 1, is obtained by adding the times T1 and T2 and dividing by two. That is, the average of all times between one failure and another and its return is calculated. It is, therefore, a simple arithmetical calculation.

Generally, this indicator is associated with the reliability of assets or asset systems. In general, the higher the MTBF the better, or fewer times of breaks and repairs over the analysed period (Shenkelberg F (2018)).

\section{MTBF-Reliability relationship}

Reliability is defined (IEC 60050-192) as the ability to perform as required, without failure, for a given time interval, under given conditions.

MTBF is defined by the arithmetic mean value of the reliability function $\mathrm{R}(\mathrm{t})$ :

$$
M T B F=\int_{0}^{\infty} R(t) d t=\int_{0}^{\infty} t f(t) d t \#(1)
$$

A constant (time independent) failure rate $\lambda$ is often considered for products. Assuming that the item is as-good-as-new after each repair, successive failure-free times are then independent random variables, exponentially distributed $(\mathrm{R}(\mathrm{t})$ $=\mathrm{e}^{-\lambda t}$ ) with the same parameter $\lambda$, and with mean $\mathrm{MTBF}=1 / \lambda$.

\section{MTBF: Reasons of Success}

The reason behind MTBF success is quickly explained: the constant failure rate assumption makes the conversion failure rate vs MTBF too convenient for daily use.

The acronym increases its appeal. Though it is less common, MTBF is sometimes referred to as a failure free time. The illusion of quantifying with a single parameter such important information is quite irresistible.

\subsection{The Constant Failure Rate Hypothesis}

As mentioned before, the constant failure rate hypothesis is often used.

The reasons behind this choice are multiple:

- $\quad$ products operate during their useful life period;

- electronic equipment do not wear-out;

- preventive replacement is performed when necessary so that failure rate stays constant;

- etc.

Reliability handbooks, guidelines and standards are often behind this ambiguous assumption.

US DoD-MIL-217 handbook is often recognized as the father of electronics reliability prediction. According to many, it is not applicable anymore, as it doesn't take into account the improvement made by modern electronic components. Further, it doesn't consider environmental constraints which have been demonstrated to influence reliability performance. The result is inaccurate (often pessimistic) evaluation of components reliability. Modern standards claim to have solved this issue. However, the wrong constant failure rate assumption is still present in modern reliability handbooks.

Let's consider one of the latest reliability handbooks: the IEC TR 62380. It has recently been superseded by IEC 61709 v3. However they are based on similar components reliability models.

The above mentioned handbook makes use of the constant failure rate assumption: "Failure rates are assumed to be constant either for an unlimited period of operation (general case) or for limited periods: in these particular cases the laws governing failure rates versus time have not been adopted in the interests of simplicity. Apart from a few exceptions, the wear-out period is never reached by electronic components. [...]NOTE: As before, and in the interests of 
simplicity, this handbook does not give the wearout failure mathematical model (for which the failure rate increases over time), but a period during which the rate can be considered constant (in some cases the period at $10 \%$ of the cumulative failure rate).

That is, only for some components showing wear-out phenomena after a certain amount of time, the life expectancy calculation model is provided. If we stay within the specified lifetime range, failure-rate can be considered constant. Limited life expectancy components are: Power transistors (for cyclic operation applications) Opto-couplers, electromechanical relays, capacitors, light emitting diodes, laser diodes, non-solid electrolyte capacitors, several relay types, switches and connectors.

Therefore, for all the remaining components we would expect a constant failure rate along their whole life.

\section{Most Common Errors about MTBF}

Among the improper uses of MTBF, following listed are the most common errors to be avoided:

Constant failure rate assumption during the whole life duration of an item is rarely applicable. MTBF use should be limited to those few cases. MTBF solely value doesn't provide any information about an item's reliability. Field failure data can be hardly described by MTBF;

(ii) MTBF is not a duration and doesn't represent the time an item will operate without failures;

(iii) MTBF is not the same as the life expectancy of an item.

\subsection{Constant Failure Rate}

In a recent study, the failure model of a railway signalling application product has been investigated in order to identify the best preventive maintenance policy aimed to reduce asset life cycle cost.

Failure data from infrastructure manager and repair warehouse have been collected, filtered and analysed.

The concerned product is an old designed piece of equipment. The rude circuitry is mainly made up by passive components, with, according to the above mentioned standard, constant failure rate within unlimited period of operation. We would therefore expect its failure rate to be constant over time and no wear-out signs to be found.

Cumulated failures and TTF information have been reported on a Weibull chart.

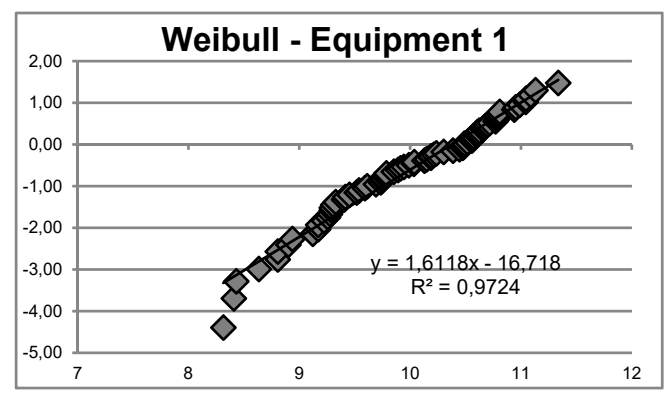

Fig. 2. Weibull chart of equipment 1

A rough graphic approach quickly helps understanding that the form (beta) factor is far from assuming ' 1 ' value. Consequently, the hypothesis of constant failure rate, proper of exponential distribution, can't be retained.

Despite of this, field data is often analysed as this hypothesis is true.

Let's consider another real case application where an electronic board used in railway signalling application failure data along almost 10 years is analysed. Fig. 3 shows failures over time for the above-mentioned board.

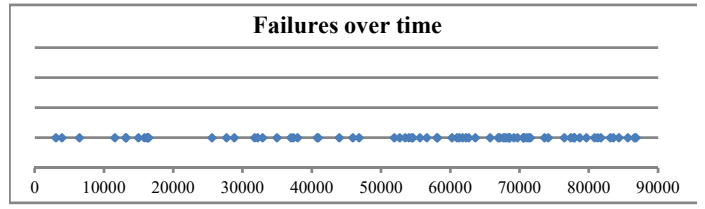

Fig. 3. Failures over time

A visual analysis reveals how the failure distribution is far from being uniform. MTBF value alone doesn't provide any information about products reliability over time.

\subsection{Failure free time}

MTBF metric is mostly used under the assumption of constant failure rate hypothesis. Though, even when this hypothesis is valid, MTBF use is often mistaken.

Let us consider the hypothesis of constant failure rate to be acceptable. The concerned item reliability can be thus described by an exponential function with constant failure rate $\lambda=1 / \mathrm{MTBF}$. In this distribution, the MTBF time corresponds to $63.2 \%$ probability of failure. In other words, if an item with a given MTBF is put in operation, there is a $63 \%$ probability that it will fail before its operating time reaches a value equal to its MTBF.

Despite this, often the term MTBF is used to denote the average operating time before an item fails (failure free time).

\subsection{MTBF vs Life expectancy}


Typical railway signalling system call for tenders, specify requirements similar to following one: "The design life of signalling system shall be a minimum of 30 years".

The above mentioned requirement clearly refers to the life duration of the equipment. In other words: "For how long the system can operate without the need for a massive overhaul?"

When limited lifetime components are used, preventive replacements are planned in order to renovate the installed material and extend the life duration of the system up to the specified value e.g. average life duration of a PC is around 5 years. If a PC with 10 years life duration is required, we need to plan an overhaul after 5 years use to replace wear parts (keyboards, mouse), upgrade RAM memory and extend storage capacity (HDD, SSD).

Too often, vendors do answer this question by providing an MTBF value and vice versa; MTBF requirements are answered by providing items life expectancy.

Though, life expectancy doesn't tell anything about item's reliability and item's reliability can't answer questions about life expectancy.

The only relationship between the life duration of an item and its reliability is the so-called bathtub-curve (Fig. 5.).

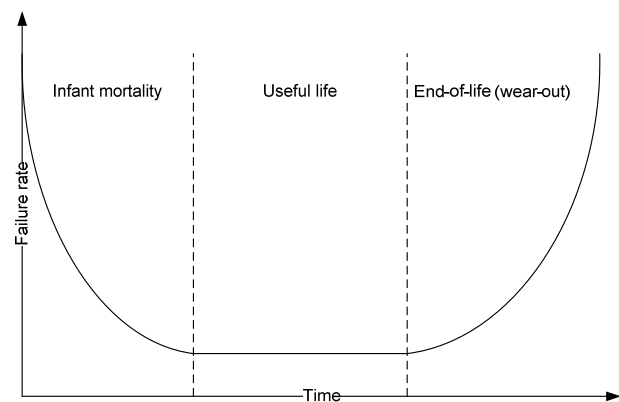

Fig. 5. Bathtub curve (hypothetical failure rate vs time)

In general, three phases are distinguishable during such a lifetime (or "bathtub") curve:

- An initial phase where residual (design) problems ("early failure" or "infant mortality") lead to relatively high-but decreasing-failure rate

- A plateau over a longer time with a more or less constant failure rate

- An increase of the failure rate at the end of life of the components ("tear and wear")

That is, reliability model is strictly linked to lifetime distribution, but, their quantitative assessments are not: hence, poor reliability is not necessarily linked to a long life expectancy and vice versa.
As already mentioned MTBF use should be as far as possible limited to cases where constant failure rate assumption is acceptable. Hence, MTBF use should be used only during useful life period (flat part of the bathtub curve). But MTBF doesn't give any indication about the duration of the useful life period. Furthermore, life expectancy is not the same as useful life.

When an assessment of the life expectancy of a product is asked the first thing should be asked back is: "What the end-of-life conditions are?"

End-of-life condition could be specified as the moment where the reliability of the item goes below a given threshold or as the moment where the failure rate of the item starts to rise (end of the flat zone of the lifetime period) and reaches a given value, or as the moment where the wear-out of the item has as affect the loss of the item's main function.

In railways, there are many examples of high reliability and short life expectancy.

LED Railway signals are normally characterised by very low failure rate values (high MTBF under the hypothesis of constant failure rate). High-brightness LEDs tend not to burn out rapidly. Rather, they slowly fade away. A carefully designed LED lighting system can see the light source last for around 50,000 to 70,000 hours (6 to 8 years) before the illumination is no longer sufficient for the intended job. But, as already mentioned MTBF is simply the probability that one item fails in a given time.

However, we could define differently the threshold "the illumination is no longer sufficient for the intended job" and instantaneously the life expectancy would be extended the probability to fail being the same or even worse as the wear-out phenomena would be more pronounced when close to the end-of-life limit.

The knowledge of lifetime distribution is a fundamental prerequisite to life expectancy assessment.

In conclusion, life expectancy evaluation is complementary to item's reliability or failure intensity evaluation. Moreover, and especially for items subject to wear-out and, therefore, limited life expectancy, MTBF is by far the less useful metric. Any use of this metric should be followed by very clear statement about the period of applicability. In these cases the use of MTBF has to be strongly discouraged, also in the light of its frequent misinterpretation as duration.

\section{MTBF vs Reliability decision making}

\subsection{Problem Statement}

We want to boost the business of our young railway company by participating to the railway 
showroom that takes place every month (excluding July and august) and lasts one week.

We are willing to buy a diesel generator to power up electric equipment of our stand.

The use of this generator is for 168 hour (1 week) run and we'd like to maintain a relatively high reliability over each exposition.

The manufacturers we are considering claims his product has 500 hours MTBF. We ask the supplier to provide us with the failure data of the last endurance test performed on the concerned product: 10 units have been tested and the times to failure (in hours) are as follows $\{1,25,45,60$, $122,517,750,1000,1250,1500\}$.

\subsection{MTTF based decision}

The classic way to calculate MTTF point estimate is to sum up the times to failure and divide by the number of failures (this is only applicable if at least one failure has occurred). We have a sum of 5,270 hours and 10 failures; MTTF point estimate is therefore 527.0 hours. This is above the vendor's claim of 500 so we are supporting the notion these are good generators.

After a quick inspection of the failure data provided by the supplier, we realize that a cluster of early failures exist which could reveal a variable decreasing failure rate of the product concerned. Thus, our assumption of constant failure rate and consequent use of MTTF metric may be wrong.

We decide to fit a Weibull distribution to the data. For a Weibull 2-parameter distribution we find beta $=0.44$ and eta $=328.37$. The data has a beta below 1 thus shows a decreasing failure rate over time.

The MTTF of our Weibull distribution is 868 hours. This gives us even more confidence that product performance is well above the vendor's claim of 500 hours MTTF. We have been confirmed that this product perfectly fits our needs.

\subsection{Reliability based decision}

As said, we use the generator for 168 hours at a time. What is the probability a generator will survive 168 hours once installed? Using the exponential distribution (MTTF point estimate) we find the reliability at 168 hours is:

$$
R(t)=e^{-\frac{t}{M T T F}} \rightarrow e^{-\frac{168}{527}}=73 \% \#(2)
$$

As during the last showroom in December the most important Railway operators will participate, we are particularly interested into knowing what is the chance of successful operation over 168 hours the 10th time we run the generator (from 1,512 to 1680 hours of life time operation). This is done by assuming the generator has survived through 9 runs (1512 hours). In this case, we find, not surprisingly given the assumed constant failure rate and memoryless property of the exponential distribution, the expected reliability is still $73 \%$.

In fact, let $\mathrm{X}$ be exponentially distributed with parameter $\lambda=1 / \mathrm{MTTF}$;

$$
\begin{aligned}
& P(X>1512+168 \mid X>1512) \\
& \frac{P(X>1680 \text { and } X>1512)}{P(X>1512)} \#(3)
\end{aligned}
$$

If $X>1680$, then $X>1512$ is redundant, so we can simplify the numerator.

$$
\begin{gathered}
P(X>1680 \mid X>1512)=\frac{P(X>1680)}{P(X>1512)} \\
=\frac{e^{-\frac{1680}{527}}}{e^{-\frac{1512}{527}}}=e^{-\frac{168}{527} \#(4)}
\end{gathered}
$$

The probability of an exponential random variable exceeding the value " $t+s$ " hours given $t$ is the same as the variable originally exceeding that value $s$, regardless of $t$.

Using the Weibull distribution we find the reliability from time 0 till 168 hours is:

$$
R(t)=e^{-\left(\frac{t}{\eta}\right)^{\beta}} \rightarrow e^{-\left(\frac{168}{328.37}\right)^{0.44}}=47 \% \#(5)
$$

The result is much lower than the estimate based on the MTTF calculation. We could make a decision based on the 868 hours MTTF value or the estimate of a $50 \%$ survival rate over the first 168 hours. $50 \%$ is not high reliability, yet 868 hours seems rather high (if compared to the 168 hours mission duration).

The reliability of the generator at the $10^{\text {th }}$ run, considering Weibull distribution and assuming it has survived during 9 runs (or 1,512 hours), is:

$$
\begin{array}{r}
P(X>1680 \mid X>1512)=\frac{P(X>1680)}{P(X>1512)} \\
=\frac{e^{-\left(\frac{1680}{328.37}\right)^{0.44}}}{e^{-\left(\frac{1512}{328.37}\right)^{0.44}}}=91 \% \#(6)
\end{array}
$$

The reliability over the 10 th run is $91 \%$. Much higher if compared to the MTTF based estimate.

Hence, this example demonstrated that decision based on MTTF, especially when considering constant failure rate model, could hide a considerable risk.

On the other hand, the use of reliability and correct reliability model not only reduces the risks 
of taking wrong decision but also gives us the means of making the best use of our assets. In this example, for instance, we could ask our supplier to apply a burn-in test to the generators and sell us one of those survived to the test. This would give us better chance to avoid a defecting item.

\section{The reasons of MTBF popularity resilience}

MTBF use, under the assumption of constant failure rate, entails a great simplicity of calculation and reliability assessment that vendors are resilient to drop. On their side, Clients do their best to obstacle the diffusion of more meaningful and proper metrics.

Here are some examples of some "Call for Tenders" RAM requirements extract from concrete railway use-cases:

1)

"For each material constituting the Signalling system, the manufacturer must guarantee at least the following reliability objectives (MTBF values):

- Workstation (PC, screen, hard disk, etc ... excluding keyboard and mouse):> 40,000 hours;

- Network hardware (switch, router, etc.): > 100,000 hours;

- Electronic and electrical equipment (power supply, UPS, etc. excluding battery): > 40000 hours;

- Electromechanical equipment (motor, circuit breaker, relay, etc.): > 50,000 hour.

We, the Vendors, attempt to provide just the right information at the right time to permit moving forward with a project.

Development team focuses on delivering new designs on time and within budget with little more than cursory review of the few field issues they have heard about at some point.

\section{Conclusions}

This paper aimed at presenting the main misunderstandings related to the concept of reliability. Despite reliability is a wide topic, it is common habit to summarize it with synthetic metrics such as MTBF. The reasons explaining the success of the MTBF are indeed related to the attempt of describing complex behaviours by means of only one metric. As explained, the abuse of MTBF, especially in railways signalling domain, is linked to two main causes:

- The presence of reliability handbooks used as a reference to demonstrate requirements in terms of reliability are met;

- The shortcut of the unverified hypothesis of constant failure rate describing the system under analysis.
The inadequacy of this metric has been evidenced by means of practical examples aiming at demonstrating that systems with high MTBF can be associated to low reliability behaviours. The stress of all these points flows into general misunderstandings from both vendors and customer's points of view.

This article is a call to action for the persons involved in reliability demonstrations. It can help reviewing hypothesis and ways of working for those who use (and abuse) MTBF when demonstrating reliability. It also helps reliability practitioners that do not use MTBF to endorse their approach in front of customers not having clear in mind the mind traps related to the abuse of MTBF.

\section{References}

Birolini, A. (2007). Reliability EngineeringTheory and Practice, Fifth Edition

IEC (2004). TR 62380, Reliability data handbook - Universal model for reliability prediction of electronics components, PCBs and equipment IEC (2004).

Mahboob, Q., Zio, E. (2018). Handbook of RAMS in Raylway Systems - Theory and Practice. CRC Press - Taylor \& Francis Group.

Pascale E, (2017). Application of the Weibull distribution for the optimization of maintenance policies of an electronic railway signaling system. ESREL 2017, European Safety and Reliability Conference.

Shenkelberg F. (2017). High MTBF with Low Reliability, nomtbf.com

Shenkelberg F. (2018). A Series of Unfortunate MTBF Assumptions, nomtbf.com

Shenkelberg F. (2018). We Need to Try Harder to Avoid MTBF, nomtbf.com

U.S. DoD (1991). MIL-HDBK-217F 